\title{
Ergonomic evaluation of postural stress in school workshop
}

\author{
Adila Md Hashim, Siti Zawiah Md Dawal and Nukman Yusoff
}

Centre of Product Design and Manufacture (CPDM), Department of Engineering Design and Manufacture, Engineering Faculty, University of Malaya, Malaysia

\begin{abstract}
The objective of this study is to compare the evaluation of postural analysis between a self-report questionnaire and physical assessments methods for students aged 13 to 15 years old in school workshop. 336 students were volunteered as participants to fill in the questionnaire and being observed in the workshop. Total of 104 positions were selected and analyzed while students performing their tasks. Questionnaire data was examined to specify the prevalence of postural stress symptoms. The relationship of postural stress by physical assessment methods (RULA and REBA methods) was defined to identify the risk level of students' working posture. From the results, comparison of four factors categorized from total of 22 questions among ages, the mean values were lower for 13 years old students meaning that they were faced higher posture problems while using the workstation. The obtained results from both physical assessment methods and questionnaire analysis have identified 13 years old students faced higher risk exposure. Analysis results emphasized the fact that self-reports questionnaire method has almost accurate as postural evaluation methods to identify physical risks in workplace. The result also shows that an intervention is needed to overcome the posture problems.
\end{abstract}

Keywords: working posture, self-reported questionnaire, physical assessment method, school workshop, student

Corresponding author email: adilamdhashim@yahoo.com.my

Phone no.: 603-79675382, Fax no.: 603-79675330 


\section{Introduction}

Concern for musculoskeletal problem in children and adolescent, many researchers has studied variety of risk factors that can lead to back pain and muscle strain. School related factors which have to do with backpack and school furniture have been identified as a common risk of back pain $[5,10]$. A study by Murphy et al. revealed that characteristics of school furniture have the highest prevalence of relationship with pain [11]. Conventional workstations that are currently used in school often described as unsuitable for schoolchildren [6, 12].

Therefore, this study conducted on school workshop to identify risk factors and quantify the postural stress. In order to analyze their working posture, this study uses two physical evaluation methods. The methods are Rapid Entire Body Assessment [3] and Rapid Upper Limb Assessment [8]. The other instrument is self report questionnaire to assess the occurrence of MSD from subjects' perception. This research also sought the prevalence of MSD among students while performing a woodworking project.

\section{Background}

Design and Technology subject named Integrated Living Skills is taught to 13 to 15 years old students in Malaysian school system as a practical experienced in performing basic hand-on machining and fabricating work [1]. Students use the school workshop for one hour and 45 minutes per week. For subject's coursework, students need to design and produce a product consists of woods and composite materials. There are two main tasks done at two different workstations which are materials cutting and assembly task. Cutting task involves of materials such as dressed woods, MDF board, and PVC pipe being cut using jigsaw machine or handsaw. The task was done at the workstation or other places for example wooden stool and desk. While assembly task included materials measurement, assembling and finishing using rasp and sand paper at the workstation. The objective of this study was to compare between ergonomic risk assessment methods and self report questionnaire in order to identify risk level of students' working posture in school workshop.

\section{Methodology}

\subsection{Workstation and subjects descriptions}

Work study was made during actual work in four school workshops at a rural secondary school in Klang district in
Selangor, Malaysia. The workstations used by students are almost similar with one another. The project's tools and materials were provided by the school administrative. The workshop can hold approximately 25 students per class. Most of the workshops have six workstations shared by four to five students per workstation. Each workstation consists of a workbench with bottom storage and stools for each students. Some students performed cutting task at different workstation such as desk and wooden stool. 336 students were randomly selected to answer the questionnaire in this study. Images were taken every 30 seconds and 104 images of most happened working postures during tasks performance were picked. All subjects were in voluntary basis and have been told about the purpose of the study. The researcher collected raw data in five months beginning of March 2011 until July 2011. This study was approved by Malaysian Ministry of Education. Demographic data of the subjects were the height range was $1.35-1.80 \mathrm{~m}$ (mean $=1.56 \mathrm{~m}, \mathrm{SD}=$ $0.074)$. The weight range was $25-101 \mathrm{~kg}$ (mean $=47.39$ $\mathrm{kg}, \mathrm{SD}=12.53)$. Body mass index $(\mathrm{BMI})$ range was 11.89 $-37.55($ mean $=19.44, \mathrm{SD}=4.34)$.

\subsection{Self report questionnaire.}

Questionnaire designed is based of Dutch Musculoskeletal Questionnaire (DMQ) [4]. The questionnaire was modified in such form to fit in the work condition. There were 22 questions and categorized into four factors, which are dynamic loads (cutting tasks), dynamic loads (assembly tasks), and workspace condition and force exertion. The dynamic loads questions covered awkward postures while performed tasks. The workspace condition included area comfort and the force exertion was about how they felt while performed cutting task. Questionnaire was given to students' right after they finished the class and was collected the next day. They were asked to answer in dichotomous scale for $1=$ Yes and $2=$ No.

\subsection{Postural analysis}

Working postures for this study were analyzed by the following methods:

- REBA assessment is suitable for whole body evaluation and best for both static and dynamic works. There are five levels of actions to indicate the obtained scores. Table 1 shows the actions level for REBA scores.

- RULA assessment is more towards upper side of the body. It is best for sedentary and seated works. There are four levels of actions to indicate the obtained scores. Table 2 shows the actions level for RULA scores. 
Table 1

\begin{tabular}{|ccc|}
\hline Score & Risk level & REBA indication \\
1 & Acceptabl & Unnecessary \\
$2-3$ & Low & May be necessary \\
$4-7$ & Medium & Necessary \\
$8-10$ & High & Necessary soon \\
$11-15$ & Very high & Immediately \\
\hline
\end{tabular}

Table 2 RULA indication

\begin{tabular}{|cc|}
\hline Score & Indication \\
$1-2$ & Posture is acceptable. \\
$3-4$ & Investigation is needed and \\
anges may be required. \\
$5-6$ & Investigation and changes are \\
& iuired soon. \\
$7<$ & Investigation and changes are \\
& luired immediately.
\end{tabular}

This study needs both methods because the tasks involved require the students to be in sitting and standing positions. Both methods will undergo statistical correlation test to identify their significant relationship between each other.

\section{$4 \quad$ Result and discussion}

\subsection{Questionnaire analysis}

Figure 1 showed the comparison of three factors among ages, the mean values were almost the same indicated they had the same awkward posture problems while using the workstation. It can be signified that 13 years old students had more difficulties in fitting themselves to the current workstation except for workspace condition factor. The lower the mean, the more risk exposure. For cutting task, 13 years old students also have highest force exertion among all ages because they used conventional handsaw instead of jigsaw machine like older students. Machine appliances topic was only covered for 14 and 15 years old students [18]. A Kruskal-Wallis test was done and showed a significant difference among ages for all factors $\left(\chi^{2}=\right.$ $8.08, p=.018$ for cutting task, $\chi^{2}=6.39, p=.041$ for assembly task, $\chi^{2}=21.0, \mathrm{p}<.05$ for workspace condition and $\chi^{2}=25.98, \quad \mathrm{p}<.05$ for force exertion).

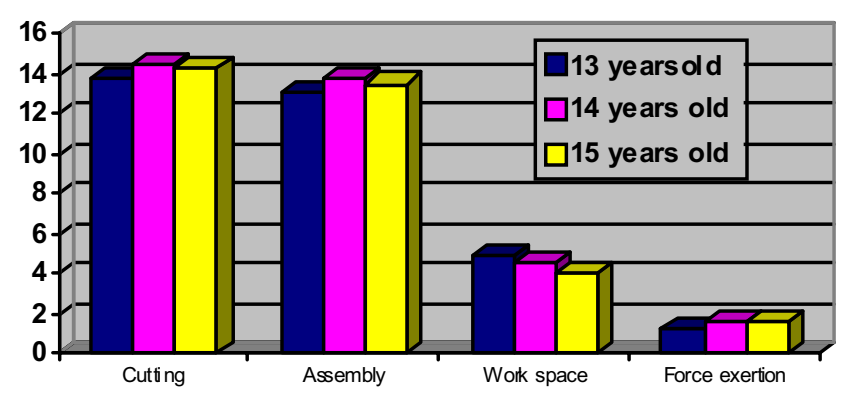

Figure 1.Mean score of all factors

\subsection{Physical assessment analysis}

Figures 2 and 3 showed the percentage of scores obtained from the evaluation. Based on the result, 13 years old students have greatest scores for both cutting and assembly task of both methods. RULA mean value were 5.4 (SD 1.13), 5.1 (SD 1.14) and 4.52 (SD 0.82) while REBA mean value were 6.0 (SD 1.54), 5.5 (SD 1.50) and 4.8 (SD 1.43) for 13, 14 and 15 years old respectively. Score 5 in RULA required changes soon while score 4, changes may be required. But in REBA, score range from 4 to 7 indicated medium level that actions are necessary. A Kruskal-Wallis test was done and showed a significant difference between ages and task group for both methods (RULA, $\chi^{2}=9.28, p=0.01$ and REBA $\chi^{2}=9.30, p=0.01$ ). For correlation test, there was a strong relationship between RULA and REBA methods which indicated both methods were reliable to each other and given the same results to identify postural stress. 


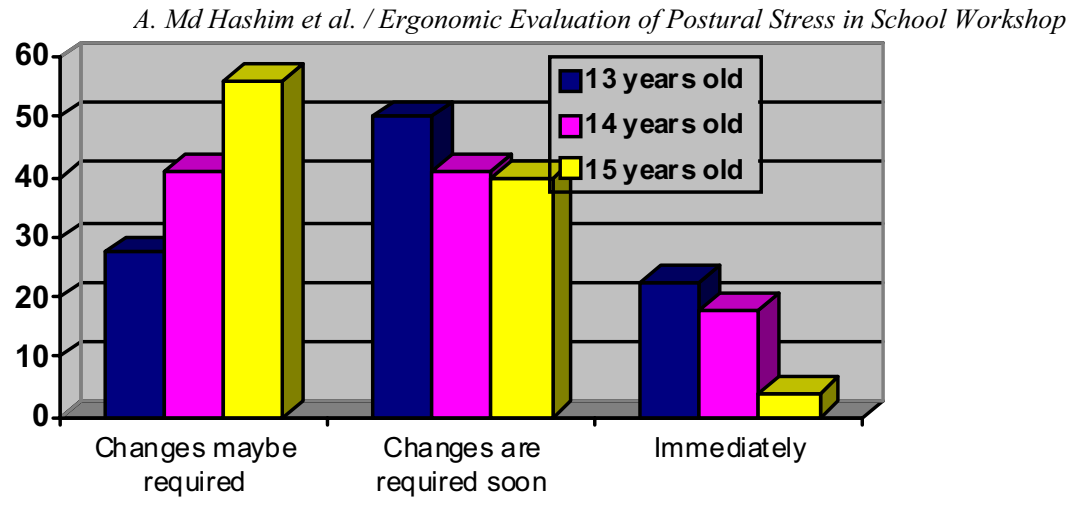

Figure 2.RULA analysis

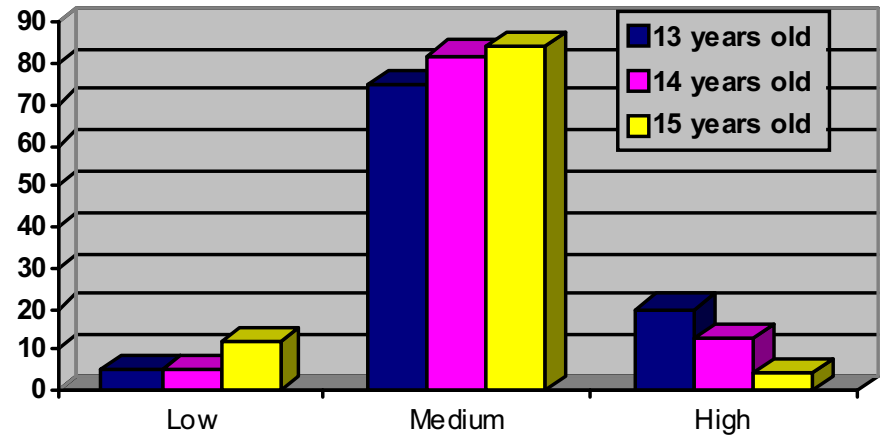

Figure 3.REBA analysis

\section{$5 \quad$ Conclusion}

In surveillance context of physical risk exposure activities, the physical assessment methods have given the same results like the questionnaire which 13 years old students have the highest risk exposure towards the current workstation. Both dynamic workloads for cutting and assembly tasks have identified those younger students cannot fit themselves to the current workstation. They also have lower mean in force exertion factor since they used conventional handsaw for cutting task.

Based on RULA and REBA scores, it has been defined that bigger students can adapt themselves towards the current workstation. Results suggested that most likely the workshop furniture tends to suit for bigger size students. The school's management maybe equipped the school workshop with adult size furniture that is unsuitable for younger students.

Participatory ergonomic action is suggested to reduce the students' postural stress as mentioned in postural assessment indication. Two types of interventions recommend are workstation modification and ergonomic education for back care.

Workstation modification may involve of suitable furniture size to tailor with students' variety sizes. In this case, the chair or stool used by students can be adjustable in height to collaborate with different body dimensions since the workbench were shared by a group of students. Other aspect of comfort like leg space, footrest and workspace envelope should be considered in redesigning of workstation. According to Linton et al. workstation modification contribution cannot totally improved students' posture [7]. Additionally, study done by Shinn et al. signified that promotion of correct body mechanic in educational ergonomic can reduced the risks of musculoskeletal injuries $[2,13]$.

\section{References}

[1] Centre of Curriculum Development (2002). Secondary School Syllabus : Integrated Living Skills. M. o. E. Malaysia.

[2] Geldhof, E., D. D. Clercq, et al. (2006). Effects of a twoschool-year multi-factorial back education program in elementary schoolchildren. IEA World Congress on Ergonomics. Maastricht.

[3] Hignett, S. and L. McAtamney (2000). "Rapid Entire Body Assessment (REBA)." Applied Ergonomics 31: 201-205.

[4] Hildebrandt, V. H. (2001). Prevention of work related musculoskeletal disorders: setting priorities using the standardized Dutch Musculoskeletal Questionnaire. Faculty of Medicine. Amsterdam, Free University of Amsterdam.

[5] Kemper, C. and R. Tholen (2004). Pain in the back: Avoiding back pain in children and teenagers. L. R. f. t. E.-f. Project. 
[6] Koskelo, R., K. Vuorikari, et al. (2007). "Sitting and standing postures are corrected by adjustable furniture with lowered muscle tension in high-school students." Ergonomics 50(10): 1643-1656.

[7] Linton, S. J., A.-L. Hellsing, et al. (1994). "The effects of ergonomically designed school furniture on pupils' attitudes, symptoms and behaviour." Applied Ergonomics 25(5): 299-304.

[8] McAtamney, L. and E. Nigel Corlett (1993). "RULA: a survey method for the investigation of work-related upper limb disorders." Applied Ergonomics 24(2): 91-99.

[9] Ministry of Education, M. (2002). SECONDARY SCHOOL SYLLABUS.INTEGRATED LIVING SKILLS.

[10] Mohd Azuan K., Zailina H., et al. (2010). "Neck, shoulder, upper back and lower back pain and associated risk factors among primary school children in Malaysia." Applied Sciences 10(5): 431-435.
[11] Murphy, S., P. Buckle, et al. (2003). Back Pain Amongst Schoolchildren And Associated Risk Factors. International Ergonomics Association XVth Triennial Congress, Seoul, Korea.

[12] Saarni, L., C.-H. Nygård, et al. (2009). "Comparing the effects of two school workstations on spine positions and mobility, and opinions on the workstations - A 2-year controlled intervention." International Journal of Industrial Ergonomics 39: 981-987.

[13] Shinn, J., K.-A. Romaine, et al. (2002). "The effectiveness of ergonomic intervention in the classroom." Work 18: 67-73. 